Pesq. Vet. Bras. 35(7):613-619, julho 2015 DOI:10.1590/S0100-736X2015000700003

\title{
Detecção de resistência às fluoroquinolonas em Campylobacter isolados de frangos de criação orgânica ${ }^{1}$
}

\author{
Beatriz S. Frasão ${ }^{2 *}$, Luana R. Côrtes ${ }^{3}$, Elmiro R. Nascimento ${ }^{4}$, Nathalie C. Cunha ${ }^{4}$, \\ Virginia L. Almeida ${ }^{4}$ e Maria Helena C. Aquino ${ }^{4}$
}

\begin{abstract}
Frasão B.S., Côrtes L.R., Nascimento E.R., Cunha N.C., Almeida V.L. \& Aquino M.H.C. 2015. [Detection of fluoroquinolone resistance in Campylobacter strains from organic poultry.] Detecção de resistência às fluoroquinolonas em Campylobacter isolados de frangos de criação orgânica. Pesquisa Veterinária Brasileira 35(7):613-619. Departamento de Tecnologia de Alimentos, Universidade Federal Fluminense, Rua Vital Brasil Filho 64, Niterói, RJ 24230-340, Brazil. E-mail: beatrizfrasao@id.uff.br

Studies have shown that resistance to quinolones in Campylobacter strains is related with Threonine-86-Isoleucine mutation. In order to investigate the presence of this mutation in sensitive and resistant Campylobacter strains to ciprofloxacin and enrofloxacin, the cecal contents of 80 broilers from organic raising chickens, slaughtered under State Inspection Service (S.I.S) of the State of Rio de Janeiro, were collected and tested for the presence of Campylobacter. The determination of ciprofloxacin and enrofloxacin susceptibility was done by disk diffusion and agar dilution methods for determining the Minimum Inhibitory Concentration (MIC). The detection of mutation in Quinolone Resistance Determinant Region (QRDR) in gyrA gene was done by sequencing. Campylobacter was isolated from $100 \%$ of the samples, being $68.75 \%$ C. jejuni and $31.25 \%$ C. coli. By the disk diffusion method, resistance to ciprofloxacin was observed in all isolates and $56.25 \%$ of the strains were resistant to enrofloxacin. By agar dilution method, all strains were resistant to ciprofloxacin (MIC $\geq 16 \mu \mathrm{g} / \mathrm{mL}$ to $\geq 64 \mu \mathrm{g} / \mathrm{mL}$ ) and full and intermediate resistance to enrofloxacin was detected in $42.50 \%$ (MIC $\geq 4-32 \mu \mathrm{g} / \mathrm{mL}$ ) and $38.75 \%$ (MIC $=2 \mu \mathrm{g} / \mathrm{mL}$ ) of the strains, respectively. Mutation Thr-86-Ile was observed in $100 \%$ of the isolates investigated. In addition to this mutation, others no silent mutations (Val-73-Glu, Ser-114-Leu, Val-88-Asp, Ala-75Asp, Gly-119-Ser, Arg-79-Lys) and silent mutations (His-81-His, Ser-119-Ser, Ala-120-Ala, Phe-99-Phe, Ala-122-Ala, Gly-74-Gly, Ile-77-Ile, Ala-91-Ala, Leu-92-Leu, Val-93-Val, Ile-106Ile, Thr-107-Thr, Gly-113-Gly, Ile-115-Ile, Gly-110-Gly) were detected. All the enrofloxacin-sensitive strains by the phenotypic methods had the Thr-86 to Ile substitution, which suggests other mechanisms contributing to enrofloxacin resistance in Campylobacter.
\end{abstract}

INDEX TERMS: Sequencing, Quinolone Determine-Resistance Region of gyrA gene, Minimum Inhibitory Concentration.

RESUMO.- Estudos têm revelado que a resistência às quinolonas em cepas de Campylobacter está relacionada à presença da mutação Treonina-86 para Isoleucina. Com o

\footnotetext{
${ }^{1}$ Recebido em 2 de dezembro de 2014.

Aceito para publicação em 27 de junho de 2015.

${ }^{2}$ Departamento de Tecnologia de Alimentos, Universidade Federal Fluminense (UFF), Rua Vital Brazil Filho 64, Niterói, RJ 24230-340, Brasil. *Autor para correspondência: beatrizfrasao@id.uff.br, beatrizfrasao90@gmail.com

${ }^{3}$ Faculdade de Medicina Veterinária, Universidade Federal Fluminense (UFF), Rua Vital Brazil Filho 64, Niterói, RJ 24230-340.

${ }^{4}$ Departamento de Saúde Coletiva Veterinária e Saúde Pública, UFF, Rua Vital Brazil Filho 64, Niterói, RJ 24230-340.
}

objetivo de investigar a presença dessa mutação em cepas de Campylobacter sensíveis e resistentes à ciprofloxacina e enrofloxacina, o conteúdo cecal de 80 frangos de corte de criação orgânica, abatidos sob Serviço de Inspeção Estadual (S.I.E.) do Estado do Rio de Janeiro, foram coletados e investigados para a presença de Campylobacter. A determinação da resistência à ciprofloxacina e enrofloxacina foi feita pela técnica de difusão em disco e de diluição em ágar para determinação da Concentração Inibitória Mínima (CIM). A detecção da mutação na Região Determinante de Resistencia às Quinolonas (RDRQ) no gene gyrA foi realizada através de sequenciamento. Campylobacter foi isolado 
a partir de $100 \%$ das amostras avaliadas, sendo $68,75 \%$ correspondente à $C$. jejuni e $31,25 \%$ à $C$. coli. No teste de difusão em disco, $100 \%$ das cepas foram resistentes à ciprofloxacina e 56,25\% das cepas foram resistentes à enrofloxacina. No teste de diluição em ágar, todas as cepas foram resistentes à ciprofloxacina apresentando CIM variando de $\geq 16-64 \mu \mathrm{g} / \mathrm{mL}$, e resistência ou resistência intermediaria à enrofloxacina foi detectada em $42,50 \%$ (CIM $\geq 4-32 \mu \mathrm{g} /$ $\mathrm{mL}$ ) e $38,75 \%$ (CIM $=2 \mu \mathrm{g} / \mathrm{mL})$ das cepas, respectivamente. A mutação Tre-86-Ile, foi observada em $100 \%$ das cepas analisadas. Além dessa mutação, foram observadas outras mutações não silenciosas (Val-73-Glu, Ser-114-Leu, Val-88Asp, Ala-75-Asp, Ser-119-Gli, Arg-79-Lis) e mutações silenciosas (His-81-His, Ser-119-Ser, Ala-120-Ala, Fen-99-Fen, Ala-122-Ala, Gli-74-Gli, Ile-77-Ile, Ala-91-Ala, Leu-92-Leu, Val-93-Val, Ile-106-Ile, Tre-107-Tre, Gli-113-Gli, Ile-115-Ile, Gli-110-Gli). A observação de que cepas sensíveis à enrofloxacina pelos testes fenotípicos apresentavam a substituição Tre-86 para Ile sugere que outros mecanismos podem contribuir para a resistência à enrofloxacina em Campylobacter.

TERMOS DE INDEXAÇÃO: Sequenciamento, Região Determinante de Resistência às Quinolonas do gene gyrA, Concentração Inibitória Mínima.

\section{INTRODUÇÃO}

Campylobacter spp. estão entre as bactérias que requerem grande atenção dos serviços de saúde coletiva, pois são patogênicas para humanos e são comumente encontradas no trato gastrointestinal das aves. A maioria das infecções por este microrganismo está associada ao consumo de carne de frango e seus subprodutos, que podem ser contaminados durante o processamento (Hermans et al. 2011, Hermans et al. 2012, Wagenaar et al. 2013). Para humanos, Campylobacter jejuni é mais patogênico além de ser mais frequente do que $C$. coli, embora coinfecções também possam ocorrer (Barbour et al. 2012, Niederer et al. 2011).

Os casos de campilobacteriose em todo o mundo ultrapassaram os casos de salmonelose e shiguelose documentados (Cover et al. 2014) e Campylobacter foi o patógeno gastrointestinal mais isolado de humanos na União Européia desde 2005. Associado ao aumento de casos de campilobacterioses documentados houve também um aumento no número de cepas de Campylobacter isoladas de humanos resistentes à ampicilina, ciprofloxacina, ácido nalidíxico e tetraciclinas. 0 mesmo foi observado quanto à resistência ao ácido nalidíxico e à tetraciclina em cepas isoladas de carnes de frango (EFSA \& ECDC 2013).

A enrofloxacina é um antimicrobiano muito usado na avicultura no Brasil, sendo amplamente empregado tanto na avicultura de corte, quanto na de postura, segundo relatos do Programa Estadual de Controle de Resíduos de Medicamentos Veterinários em Alimentos de Origem Animal (PAMvet) do Estado do Paraná (Machinski Junior et al. 2005). 0 uso excessivo e sem controle de antimicrobianos na produção animal pode contribuir para seleção de bactérias resistentes, que podem ser disseminadas durante a produção ou processamento dos alimentos. Em razão disto, desde 2006, a União Européia e outras nações baniram o uso de alguns antimicrobianos na produção animal (EFSA, ECDC \& EMEA 2009).

A resistência às quinolonas em Campylobacter spp. está relacionada principalmente à mutação Tre-86-Ile na RDRQ do gene gyrA da DNA girase, de acordo com vários autores (Yang et al. 2006, Qin et al. 2011, Iovine 2013, Frasao \& Aquino 2014). Entretanto, outras mutações (Tre-86-Ile, Asp-90-Asn, Ala-70-Tre, Asp-85-Tir, Pro-104-Ser) nessa região já foram descritas como relacionadas à resistência em Campylobacter, além de mutações no gene parC, da topisomerase IV e da ação exacerbada da bomba de efluxo CmeABC devido à mutação no gene $c m e \mathrm{R}$ (Bachoual et al. 2001, Piddock et al. 2003, Yang et al. 2006, Qin et al. 2011, Wieczorek \& Osek 2013, Iovine 2013, Hungaro et al. 2015).

No Brasil, a produção e comercialização dos produtos orgânicos foram aprovadas pela Lei 10.831, de 23 de dezembro de 2003 (Brasil 2003) e sua regulamentação ocorreu com a publicação do Decreto № 6.323 em 27 de dezembro de 2007 (Brasil 2007). Segundo a lei supracitada, o sistema orgânico de produção agropecuária adota técnicas específicas, visando otimizar os recursos naturais e socioeconômicos disponíveis, e também a sustentabilidade ecológica e econômica. $\mathrm{O}$ uso de antimicrobiano como promotor de crescimento na produção de carne orgânica é proibido (Crabone et al. 2005, Brasil 2008) e tratamentos terapêuticos alternativos são realizados (Griggs \& Jacob 2005).

A criação orgânica de frangos de corte difere da criação convencional pelo fato do animal ter acesso ao ambiente, sendo uma criação "à pasto" (Rosenquist et al. 2013) e pela idade de abate ser mais avançada. Sendo assim, esses animais podem se infectar com Campylobacter presente no ambiente ou oriundo de aves silvestres. Estudos revelam uma prevalência de Campylobacter spp. em frangos de criação orgânica entre $60 \%$ a $100 \%$ (Heuer et al. 2001, El-Shibiny et al. 2005, Overbeke et al. 2006, Colles et al. 2008, Esteban et al. 2008, Hoogenboom et al. 2008, EFSA 2010). Dentro desse contexto, objetivou-se com esta pesquisa investigar a ocorrência de Campylobacter jejuni e C. coli em frangos de criação orgânica e determinar sua suscetibilidade à ciprofloxacina e enrofloxacina através de métodos fenotípicos e genotípicos.

\section{MATERIAL E MÉTODOS}

Foram coletadas 80 amostras de conteúdo cecal de frangos de corte de criação orgânica provenientes de dois lotes (40 animais por lote). A coleta foi realizada imediatamente após a evisceração, na linha de abate, em abatedouro com Serviço de Inspeção Estadual (S.I.E.) do Estado do Rio de Janeiro. Suabes contendo as fezes foram diluídos em quatro mililitros de água destilada esterilizada e $0,1 \mathrm{~mL}$ foram inoculados em placas contendo Ágar Columbia suplementado com carvão ativado $(0,4 \%)$ e suplemento seletivo CAMPYLOFAR $^{\circledR}$ (CEFAR). Paralelamente, três mililitros da diluição foi previamente filtrada em membrana milipore $(0,65 \mu \mathrm{m})$ e o filtrado foi estriado nas placas contendo Ágar Columbia suplementado com carvão ativado $(0,4 \%)$. As placas foram incubadas a $37^{\circ} \mathrm{C}$ por 48 horas, em condições de microaerofilia. As colônias suspeitas foram confirmadas pela técnica de PCR após identificação presuntiva pelas características morfotintoriais e testes de produção de oxidase e catalase. 
Para a confirmação por PCR, o DNA foi extraído com o kit de extração comercial 'Wizard ${ }^{\circledR}$ Genomic DNA Purification Kit' (PRO$\mathrm{MEGA}^{\circledR}$ ) e foi utilizada a técnica de PCR multiplex, baseada no que foi descrito por Harmon et. al. (1997) modificada por Aquino et al. (2002). Os primers pg3/pg50 que amplificam uma região conservada nas duas espécies (Campylobacter jejuni e C. coli), relacionada ao gene da flagelina (Oyofo et al. 1992), e primers C-1 /C-4 que amplificam uma região específica da espécie $C$. jejuni (Winters \& Slavik 1995) foram usados. A reação de amplificação foi feita com volume final de $50 \mu \mathrm{L}$, contendo $5 \mu \mathrm{L}$ do DNA amostral, 1X PCR Buffer (500mM KCl, 100mM Tris-HCl [pH 8.5]), $4 \mu \mathrm{L}$ $(200 \mu \mathrm{M}$ cada) dATP, dCTP, dGTP e dTTP, $0,4 \mu \mathrm{M}$ de cada primer pg3 e pg50, 0,2 $\mu \mathrm{M}$ de cada primer $\mathrm{C} 1$ e C4, 2,5 U de Taq DNA polimerase e 5,5mM/L de $\mathrm{MgCl}_{2}$. A desnaturação inicial foi realizada a $94^{\circ} \mathrm{C}$ por quatro minutos, seguida por 25 ciclos de amplificação constituídos em um minuto a $94^{\circ} \mathrm{C}$, um minuto a $55^{\circ} \mathrm{C}$, um minuto a $72^{\circ} \mathrm{C}$ e extensão a $72^{\circ} \mathrm{C}$ por sete minutos. Para a verificação dos amplicons, foi realizada eletroforese em cuba horizontal 'Eletrophoresis Cell (BioAmérica)' com TBE 0,5x, com fonte Power Pac 300 (Bio-Rad), em gel de agarose 1,5\% corado com GelRed. Os produtos da amplificação foram visualizados e fotografados em transiluminador com luz ultravioleta (Nova Instruments). Foram utilizadas como controle positivo as cepas C. jejuni ATCC 33560 e C. coli ATCC 33559, gentilmente cedidas pelo Instituto Nacional de Controle de Qualidade em Saúde da Fundação Oswaldo Cruz (INCQS/Fiocruz).

0 perfil de resistência à enrofloxacina e ciprofloxacina das cepas isoladas foi determinado pelo método de difusão em disco e pelo método de diluição em ágar, para determinar a concentração inibitória mínima (CIM) de acordo com os critérios determinados pelo "Clinical and Laboratory Standards Institute" (CLSI 2010). As concentrações utilizadas foram $64 \mu \mathrm{g} / \mathrm{mL}, 32 \mu \mathrm{g} / \mathrm{mL}, 16 \mu \mathrm{g} / \mathrm{mL}$, $8 \mu \mathrm{g} / \mathrm{mL}, 4 \mu \mathrm{g} / \mathrm{mL}, 2 \mu \mathrm{g} / \mathrm{mL}$ e $1 \mu \mathrm{g} / \mathrm{mL}$. A suspensão de Campylobacter inoculada foi ajustada ao equivalente a turbidez do padrão de McFarland 0.5 . As placas foram incubadas a $37^{\circ} \mathrm{C}$ por 48 horas, em microaerofilia.

Todas as cepas isoladas e as cepas controle $C$. jejuni ATCC 33560 e $C$. coli ATCC 33559 foram submetidas ao sequenciamento da Região Determinante de Resistência às Quinolonas (RDRQ) do gene gyrA. Os primers CjgyrA QRDR F e CjgyrA QRDR R (Parkhill et al. 2000, Price et al. 2005), específicos para amplificar a RDRQ do gene gyrA, foram utilizados. Para a reação foi utilizado $50 \mu \mathrm{L}$ contendo $5 \mu \mathrm{L}, 1 \mathrm{X}$ PCR Buffer $(500 \mathrm{mM} \mathrm{KCl}, 100 \mathrm{mM}$ Tris- $\mathrm{HCl}[\mathrm{pH}$ 8.5]), $5 \mu \mathrm{L}$ (1mM cada) dATP, dCTP, dGTP e dTTP, $0,5 \mu \mathrm{L}$ de cada primer, $1.0 \mathrm{U}$ Taq DNA polymerase e $2 \mathrm{mM} \mathrm{MgCl}_{2}$. A desnaturação inicial foi realizada a $94^{\circ} \mathrm{C}$ por cinco minutos e 35 ciclos com desnaturação por 30 segundos a $94^{\circ} \mathrm{C}$, anelamento do primer a $55^{\circ} \mathrm{C}$ por 30 segundos, extensão a $72^{\circ} \mathrm{C}$ por um minuto e extensão final a $72^{\circ} \mathrm{C}$ por 10 minutos. Para a verificação dos amplicons, foi realizada eletroforese em cuba horizontal 'Eletrophoresis Cell (BioAmérica)’ com TBE 0,5x, com fonte Power Pac 300 (Bio-Rad), em gel de agarose $1,5 \%$ corado com GelRed. Os produtos da amplificação foram visualizados e fotografados em transiluminador com luz ultravioleta (Nova Instruments). 0 produto da amplificação foi purificado com Kit de Purificação Comercial da $\mathrm{GE}^{\circledR}$, seguindo as instruções descritas no manual. A dosagem do DNA purificado foi realizada de acordo com o recomendado no protocolo "Low DNA Mass Ladder" (Invitrogen).

Para o sequenciamento foi utilizado sequenciador automático 3730XL DNA Analyzer (Applied Biosystems). As sequências foram montadas e sua qualidade foi avaliada através da obtenção dos cromatogramas das sequências-consenso utilizando o BioEdit Sequence Alignment Editor (Hall 1999) e Molecular Evolutionary Genetics Analysis Version 6.0-MEGA6 (Tamura et al. 2013). Foram comparadas as sequências da região RDRQ do gene gyrA de cepas sensíveis de $C$. jejuni (L04566.1) e C. coli (U63413.1) obtidas no GenBank com as sequências da região RDRQ dos isolados a fim de investigar a ocorrência de mutações.

\section{RESULTADOS}

Campylobacter spp. foram isolado de $100 \%$ das amostras coletadas e através da técnica de PCR multiplex, $C$. jejuni foi identificado em $68,75 \%$ e $C$. coli em $31,25 \%$ das amostras. Nos dois lotes, a espécie mais isolada foi $C$. jejuni, sendo $82,50 \%$ no lote A e 55,00\% no lote B (Quadro 1).

$\mathrm{Na}$ avaliação do perfil de resistência aos antimicrobianos no teste de difusão em disco, 100\% e 56,25\% das cepas foram resistentes à ciprofloxacina e à enrofloxacina, respectivamente. No teste de diluição em ágar, todas as cepas foram resistentes à ciprofloxacina e apresentaram CIM variando de $16 \mu \mathrm{g} / \mathrm{mL}$ a $\geq 64 \mu \mathrm{g} / \mathrm{mL}$. Para a enrofloxacina, apenas $18,75 \%$ das cepas foram sensíveis (CIM $\leq 1 \mu \mathrm{g} / \mathrm{mL}$ ), enquanto $38,75 \%$ apresentaram resistência intermediária (CIM $=2 \mu \mathrm{g} / \mathrm{mL})$, e $42,50 \%$ foram resistentes (CIM $\geq 4 \mu \mathrm{g}$ / $\mathrm{mL}$ ). No lote A, a maioria das cepas apresentou resistência à enrofloxacina pelos métodos de difusão em disco $(72,50 \%)$ e diluição em ágar $(67,50 \%)$, enquanto $7,5 \%$ apresentaram resistência intermediária. No lote B, 40,00\% e 17,50\% das cepas foram resistentes pelo teste de difusão em disco e diluição em ágar, respectivamente, enquanto 70\% apresentaram resistência intermediária (Quadro 1). A maioria $(74,07 \%)$ das cepas resistentes à enrofloxacina no teste de diluição em ágar do lote A apresentou CIM $=4 \mu \mathrm{g} / \mathrm{mL}$. A maior CIM para enrofloxacina observada em ambos os lotes foi de $32 \mu \mathrm{g} / \mathrm{mL}$, sendo que apenas uma cepa em cada lote apresentou esta concentração inibitória mínima, enquanto para ciprofloxacina foi observada CIM $\geq 64 \mu \mathrm{g} / \mathrm{mL}$.

Quadro 1. Resultados do isolamento e da suscetibilidade das cepas de Campylobacter jejuni e $C$. coli à enrofloxacina pelo método de difusão em disco e pela determinação da concentração inibitória mínima (CIM)

\begin{tabular}{|c|c|c|c|c|c|}
\hline \multicolumn{3}{|c|}{ Testes } & C. jejuni & C. coli & Total \\
\hline & Isola & mento & $33(82,50 \%)$ & $7(17,50 \%)$ & $40(100 \%)$ \\
\hline & Difusão & Resistente & $25(62,50 \%)$ & $4(10,00 \%)$ & $29(72,50 \%)$ \\
\hline & em disco & Sensível & $8(20,00 \%)$ & $3(7,50 \%)$ & $11(27,50 \%)$ \\
\hline & CIM & Resistente & $23(57,50 \%)$ & $4(10,00 \%)$ & $27(67,50 \%)$ \\
\hline & & Intermediária & $2(5,00 \%)$ & $1(2,50 \%)$ & $3(7,50 \%)$ \\
\hline & & Sensível & $8(20,00 \%)$ & $2(5,00 \%)$ & $10(25,00 \%)$ \\
\hline \multirow{6}{*}{ 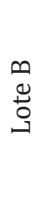 } & Isola & mento & $22(55,00 \%)$ & $18(45,00 \%)$ & $40(100 \%)$ \\
\hline & Difusão & Resistente & $8(20,00 \%)$ & $8(20,00 \%)$ & $16(40,00 \%)$ \\
\hline & em disco & Sensível & $14(35,00 \%)$ & $10(25,00 \%)$ & $24(60,00 \%)$ \\
\hline & CIM & Resistente & $4(10,00 \%)$ & $3(7,50 \%)$ & $7(17,50 \%)$ \\
\hline & & Intermediária & $15(37,50 \%)$ & $13(32,50 \%)$ & $28(70,00 \%)$ \\
\hline & & Sensível & $3(7,50 \%)$ & $2(5,00 \%)$ & $5(12,50 \%)$ \\
\hline \multirow{6}{*}{$\begin{array}{l}\text { त्ञే } \\
0\end{array}$} & Isola & mento & $55(68,75 \%)$ & $25(31,25 \%)$ & $80(100 \%)$ \\
\hline & Difusão & Resistente & $33(41,25 \%)$ & $12(15,00 \%)$ & $45(56,25 \%)$ \\
\hline & em disco & Sensível & $22(27,50 \%)$ & $13(16,25 \%)$ & $35(43,75 \%)$ \\
\hline & CIM & Resistente & $27(33,75 \%)$ & $7(8,75 \%)$ & $34(42,50 \%)$ \\
\hline & & Intermediária & $17(21,25 \%$ & $14(17,50 \%)$ & $31(38,75 \%$ \\
\hline & & Sensível & $11(13,75 \%)$ & $4(5,00 \%)$ & $15(18,75 \%$ \\
\hline
\end{tabular}

Na pesquisa de mutação na Região Determinante de Resistência às Quinolonas (RDRQ) do gene gyrA, 100\% das cepas apresentaram a mutação Tre-86-Ile. Outras mu- 
tações silenciosas (His-81-His, Ser-119-Ser, Ala-120-Ala, Fen-99-Fen, Ala-122-Ala, Gli-74-Gli, Ile-77-Ile, Ala-91-Ala, Leu-92-Leu, Val-93-Val, Ile-106-Ile, Tre-107-Tre, Gli-113Gli, Ile-115-Ile, Gli-110-Gli) foram observadas em ambos os lotes e mutações não silenciosas (Val-73-Glu, Ser-114-Leu, Val-88-Asp, Ala-75-Asp, Ser-119-Gli, Arg-79-Lis) também foram observadas em cepas isoladas dos frangos do lote B. Além das mutações por substituição citadas acima, no códon 73 foi observada mutação por deleção (GTG $\rightarrow$ G_G) em duas cepas (Fig.1).

\section{DISCUSSÃO}

Nesse estudo, Campylobacter spp. foram isolado de 100\% das amostras investigadas. Outros autores também encontraram uma alta frequência de Campylobacter em frangos de criação orgânica, variando de $66 \%$ a $80 \%$ (Cui et al. 2005, Luangtongkum et al. 2006, Soonthornchaikul et al. 2006). A elevada ocorrência de Campylobacter spp. nos frangos de criação orgânica segundo Adkin et al. (2006) é resultante da idade de abate avançada (em média 70 a
90 dias), com maior exposição das aves ao ambiente nesse tipo de criação.

No presente trabalho, todas as cepas apresentaram alto nível de resistência à ciprofloxacina quando observada a $\mathrm{CIM}$, variando entre $16 \mu \mathrm{g} / \mathrm{mL}$ a $\geq 64 \mu \mathrm{g} / \mathrm{mL}$. Para enrofloxacina, $42,50 \%$ das cepas foram resistentes (CIM $\geq 4-32 \mu \mathrm{g}$ / $\mathrm{mL}$ ) e $38,75 \%$ apresentaram resistência intermediária ( $\mathrm{CIM}=2 \mu \mathrm{g} / \mathrm{mL}$ ), demonstrando um nível de resistência inferior ao detectado para ciprofloxacina. A maior resistência à ciprofloxacina observada nesse estudo pode ser explicada pelo fato da ciprofloxacina ser considerada um metabólito da enrofloxacina (Idowu et al. 2010). Com o uso frequente da enrofloxacina na avicultura, na metabolização deste antimicrobiano, cepas resistentes à ciprofloxacina podem ter sido selecionadas.

Em criações orgânicas espera-se que não haja a utilização de antimicrobianos na produção, porém 100\% das cepas isoladas nesse estudo apresentaram resistência à ciprofloxacina. Este achado pode estar relacionado com a persistência de cepas resistentes no ambiente de criação.
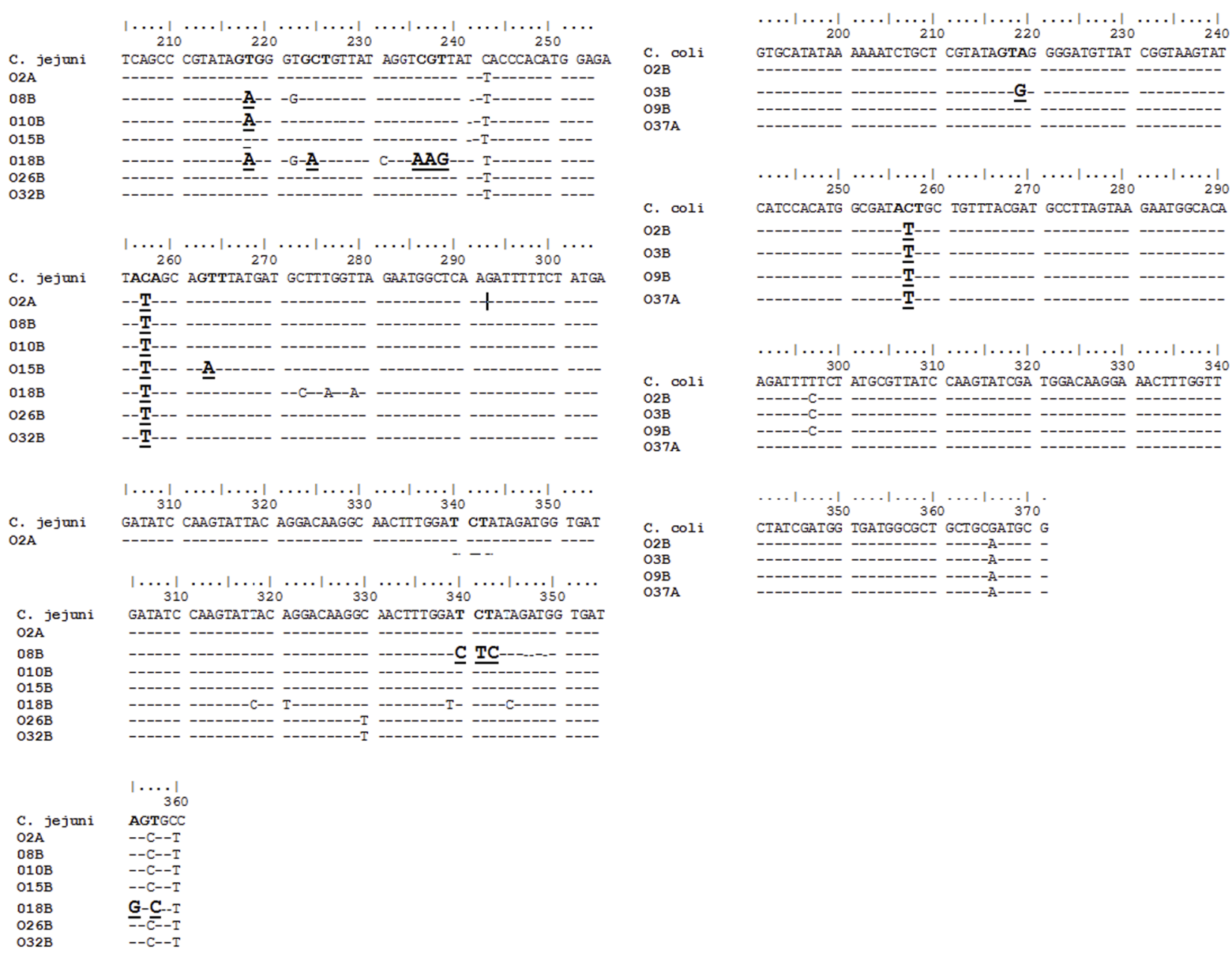

Fig.1. Representação das mutações observadas nas sequências obtidas no sequenciamento da Região Determinante de Resistência às Quinolonas (RDRQ) do gene gyrA das cepas de Campylobacter jejuni e C. coli isoladas dos frangos de criação orgânica. C. jejuni e C. coli são padrões sensíveis obtidos no GenBank (L04566.1 e U63413.1, respectivamente). 02A, 08B, 010B, 015B, 018B, 026B, 032B são cepas representantes das mutações observadas nas sequências obtidas das cepas de C. jejuni isoladas neste experimento. 02B, 03B, 09B e 37A são cepas representantes das mutações observadas nas sequências obtidas das cepas de $C$. coli isoladas neste experimento. As alterações caracterizadas como não silenciosas estão destacadas em negrito, as demais são caracterizadas como silenciosas. 
Essa persistência pode estar relacionada à produção de biofilmes nos sistemas de suprimento de água nas instalações de criação de aves como demonstrado por Reeser et al. (2007) e Reuter et al. (2010). A formação de biofilme por $C$. jejuni é aumentada sob condições aeróbicas e os biofilmes são reservatórios de células viáveis destes microrganismos, o que indica que há uma boa adaptação desse patógeno para a sobrevivência no ambiente (Joshua et al. 2006; Reuter et al. 2010). Diversos estudos revelam que a CIM de Campylobacter rapidamente aumenta após o uso de fluoroquinolonas em criações avícolas e a resistência das cepas pode se manter por várias semanas após a supressão do tratamento. Essas cepas podem persistir tanto nas aves como no ambiente de criação (Humphrey et al. 2005).

Resistência a antimicrobianos em patógenos se tornou uma preocupação em saúde coletiva e essa preocupação vem aumentando em muitos países. Os resultados obtidos nesse estudo, embora incluam apenas criações orgânicas do Estado do Rio de Janeiro, onde supostamente não se fez uso de antimicrobianos, revelam uma predominância de cepas de Campylobacter resistentes às fluoroquinolonas na criação. Em 2005, o Food and Drug Administration (FDA) nos EUA proibiu o uso das fluoroquinolonas na avicultura alegando que cepas de Campylobacter resistentes às fluoroquinolonas podiam ser transmitidas pela carne desses animais e causar infecção humana, sendo uma ameaça à saúde coletiva (FDA 2014). Além dos EUA, na Noruega e Austrália, onde também não é permitido o uso de quinolonas na avicultura, estudos realizados relatam a inexistência de cepas de Campylobacter spp. resistentes às fluoroquinolonas (Cui et al. 2005, Norström et al. 2007, Obeng et al. 2012). No Brasil, não é proibido o uso de enrofloxacina, medicamento de uso exclusivo em medicina veterinária, quando este tem fins terapêuticos em avicultura.

Nesse estudo a mutação característica no códon 86 que substitui a treonina pela isoleucina (Tre-86-Ile) foi observada em todas as cepas sequenciadas, o que justifica a resistência à ciprofloxacina detectada em todas as cepas investigadas. Qin et al. (2011) também demonstraram relação da presença da mutação Tre-86-Ile com altos níveis de resistência à ciprofloxacina, enquanto Duarte et al. (2014) encontraram a mutação em todas as cepas que apresentaram resistência à ciprofloxacina em testes fenotípicos.

No entanto, nesse estudo foi detectada a presença de mutação Tre-86-Ile em cepas sensíveis à enrofloxacina (CIM $\leq 1 \mu \mathrm{g} / \mathrm{mL}$ ). Em sua revisão, Možina et al. (2011) relatam que alguns autores afirmam que além de mutações pontuais, a resistência a antimicrobianos pode ser conferida por bombas de efluxo, que bombeiam ativamente as moléculas dos fármacos evitando a acumulação intracelular necessária para a letalidade do microrganismo. Em Campylobacter, Hungaro et al. (2015) demonstraram a presença dos três genes requeridos para sintetizar o sistema de efluxo CmeABC em $90 \%$ das cepas analisadas. 0 uso de inibidores da bomba de efluxo no referido estudo resultou em uma significante redução na CIM dos antimicrobianos testados incluindo a ciprofloxacina. Jeon et al. (2011) afirmam que a superexpressão do operon cmeGH em $C$. jejuni, relacionado ao gene cmeG que codifica um transportador na bomba de efluxo, aumentou significativamente a sua resistência às fluoroquinolonas. Cagliero et al. (2007) selecionaram um mutante de C. jejuni multirresistente através do aumento das concentrações de enrofloxacina in vitro e afirmaram que a bomba de efluxo CmeABC tem relação com a resistência neste microrganismo. A resistência à ciprofloxacina conferida por bomba de efluxo também foi caracterizada em cepas de Actinobacillus pleuropneumoniae (Wang et al. 2010).

Além da mutação Tre-86-Ile, que confere resistência às fluoroquinolonas, outras mutações não silenciosas por substituição foram detectadas em cepas do lote B porém diferentes de outras mutações já descritas na literatura como relacionadas à resistência de Campylobacter spp. às fluoroquinolonas (Wang et al. 1993, Wilson et al. 2000, Bachoual eu al. 2001, Hakanen et al. 2002, Qin et al. 2011, Iovine 2013, Wieczorek \& Osek 2013, Hungaro et al. 2015). A mutação por deleção no códon 73 (GTG $\rightarrow$ G_G) observada em duas cepas de $C$. jejuni não foi aparentemente descrita anteriormente em cepas de Campylobacter resistentes à fluoroquinolonas. Mutações silenciosas são frequentemente descritas em cepas sensíveis e resistentes às fluoroquinolonas e dentre as mutações silenciosas identificadas no presente trabalho, a mutação Fen-99-Fen foi descrita em cepas de $C$. coli resistentes a quinolonas (Qin et al. 2011), His-81-His e Ser-119-Ser foram descritas em cepas sensíveis às quinolonas (Beckmann et al. 2004) e a mutação Ala-120-Ala foi descrita em cepas sensíveis e resistentes (Wilson et al. 2000, Hakanen et al. 2002).

\section{CONCLUSÕES}

O alto nível de resistência evidenciado pela CIM observada e a alta frequência de cepas de Campylobacter resistentes às fluoroquinolonas detectadas em frangos de criação orgânica pode ser resultante do uso desses antimicrobianos na avicultura, com persistência de cepas resistentes no sistema de produção de frango de corte.

A observação da presença da mutação Tre-86-Ile no gene gyrA em cepas sensíveis à enrofloxacina sugere a participação de outros mecanismos que conferem resistência à esse antimicrobiano em cepas de Campylobacter spp.

Agradecimentos.- À doutoranda do Programa de Pós-graduação em Higiene Veterinária e Processamento Tecnológico de Produtos de Origem Animal, Marion Pereira da Costa, pelo apoio em coleta. À estagiária do Laboratório de Doenças Infecciosas da UFF, Luiza Curzio de Souza, pelo apoio em bancada. À Pró-Reitoria de Pesquisa, Pós-graduação e Inovação (PROPPI/FOPESQ). B.S. Frasao foi apoiada pela Coordenação de Aperfeiçoamento de Pessoal de Nível Superior (CAPES), e pelo Conselho Nacional de Desenvolvimento Científico e Tecnológico (CNPq).

\section{REFERÊNCIAS}

Adkin A., Hartnett E., Jordan L., Newell D. \& Davison H. 2006. Use of a systematic review to assist the development of Campylobacter control strategies in broilers. J. Appl. Microbiol. 100:306-315.

Aquino M.H.C., Filgueiras A.L.L., Ferreira M.C.S., Oliveira S.S., Bastos M.C. \& Tibana A. 2002. Antimicrobial resistance and plasmid profiles of Campylobacter jejuni and Campylobacter coli from human and animal sources. Lett. Appl. Microbiol. 34:149-153.

Barbour E.K., Ahmadieh D., Harakeh S. \& Kumosani T. 2012. Impact of antimicrobials use in chickens on emergence of drug-resistant Campylobacter organisms in humans. Int. Arab. J. Antimicrob. Agents 2(4):1. 
Bachoual R., Ouabdesselam S., Mory F., Lascols C., Soussy C.J. \& Tankovic J. 2001. Single or double mutational alterations of gyrA associated with fluoroquinolone resistance in Campylobacter jejuni and Campylobacter coli. Microb. Drug Resist. 7:257-261.

Beckmann L., Müller M., Luber P., Schrader C., Bartelt E. \& Klein G. 2004. Analysis of gyrA mutations in quinolone-resistant and -susceptible Campylobacter jejuni isolates from retail poultry and human clinical isolates by non-radioactive single-strand conformation polymorphism analysis and DNA sequencing. J. Appl. Microbiol. 96:1040-1047.

Brasil 2003. Lei no 10831, de 23 de dezembro de 2003. Dispõe sobre a agricultura orgânica e dá outras providências. Publicado no Diário Oficial da União de 24/12/2003, Seção 1, p.8.

Brasil 2007. Decreto oㅜ 6.323, de 27 de dezembro de 2007. Regulamenta a Lei no 10.831, de 23 de dezembro de 2003, que dispõe sobre a agricultura orgânica, e dá outras providências. Publicado no Diário Oficial da União de 28/12/2007, Seção 1, p. 2.

Brasil 2008. Instrução Normativa no 64, de 18 de dezembro de 2008. Aprova o regulamento técnico para os sistemas orgânicos de produção animal e vegetal. Publicado no Diário Oficial da União de 12/2009, p.21.

Cagliero C., Maurel M., Cloeckaert A. \& Payot S. 2007. Regulation of the expression of the CmeABC efflux pump in Campylobacter jejuni: identification of a point mutation abolishing the binding of the CmeR repressor in an in vitro - selected multidrug-resistant mutant. FEMS Microbiol Lett. 267:89-94.

CLSI 2010. Methods for Antimicrobial Dilution and Disk Susceptibility Testing of Infrequently Isolated or Fastidious Bacteria: Approved guideline. 2nd ed. CLSI document M45-A2. Clinical and Laboratory Standards Institute, Wayne, PA.

Colles F.M., Jones T.A., McCarthy N.D., Sheppard S.K., Cody A.J., Dingle K.E., Dawkins M.S. \& Maiden M.C.J. 2008. Campylobacter infection of broiler chickens in a free-range environment. Environ. Microbiol. 10:2042-2050.

Cover K.E., Ruiz S.A. \& Chapman A.S. 2014. Reported gastrointestinal infections in the U.S. Air Force, 2000-2012. MSMR 21:2-7.

Crabone G.T., Moori R.G. \& Sato G.S. 2005. Fatores relevantes na decisão de compra de frango caipira e seu impacto na cadeia produtiva. Org. Rurais Agroind. 7(3):312-323.

Cui S., Ge B., Zheng J. \& Meng J. 2005. Prevalence and antimicrobial resistance of Campylobacter spp. and Salmonella serovars in organic chickens from Maryland retail stores. Appl. Environ. Microbiol. 71:4108-4111.

Duarte A., Santos A., Manageiro V., Martins A., Fraqueza M.J., Caniça M., Domingues F.C. \& Oleastro M. 2014. Human, food and animal Campylobacter spp. isolated in Portugal: high genetic diversity and antibiotic resistance rates. Int. J. Antimicrob. Agents 44:306-313.

EFSA (European Food Safety Authority). 2010. Analysis of the baseline survey on the prevalence of Campylobacter in broiler batches and of Campylobacter and Salmonella on broiler carcasses in the EU, 2008, part B: analysis of factors associated with Campylobacter colonisation of broiler batches and with Campylobacter contamination of broiler carcasses; and investigation of the culture method diagnostic characteristics used to analyse broiler carcass samples. EFSA J. 8(8). 1522p.

EFSA (European Food Safety Authority), ECDC (European Centre for Disease Prevention and Control), EMEA, (European Medicines Agency). 2009. Scientific Committee on Emerging and Newly Identified Health Risks. Joint Opinion on antimicrobial resistance (AMR) focused on zoonotic infections Scientific Opinion of the European Centre for Disease Prevention and Control; Scientific Opinion of the Panel on Biological Hazards; Opinion of the Committee for Medicinal Products for Veterinary Use; Scientific Opinion of the Scientific Committee on Emerging and Newly Identified Health Risks, EFSA J. 7(11). 78p.

EFSA (European Food Safety Authority), ECDC (European Centre for Disease Prevention and Control). 2013. The European Union Summary Report on Trends and Sources of Zoonoses, Zoonotic Agents and Foodborne Outbreaks in 2011. EFSA J. 1(4). 250p.

El-Shibiny A., Connerton P.L. \& Connerton I.F. 2005. Enumeration and diversity of Campylobacters and bacteriophages isolated during the rearing cycles of free-range and organic chickens. Appl. Environ. Microbiol. 71:1259-1266.
Esteban J.I., Oporto B., Aduriz G., Juste R.A. \& Hurtado A. 2008. A survey of food-borne pathogens in free-range poultry farms. Int. J. Food. Microbiol. 123:177-182.

FDA 2014. Enrofloxacin for Poultry. Food and Drug Administration. Disponível em <http://www.fda.gov/AnimalVeterinary/SafetyHealth/Recalls Withdrawals/ucm042004.htm> Acesso em 21 nov. 2014.

Frasão B.S. \& Aquino M.H.C. 2014. Campylobacter spp. em aves (Gallus gallus domesticus) e suínos (Sus domesticus): resistência a antimicrobianos e importância na saúde coletiva. Encicl. Biosf. 10(18):744-758.

Griggs J.P. \& Jacob J.P. 2005. Alternatives to antibiotics for organic poultry production. J. Appl. Poult. Res. 14(4):750-756.

Hakanen A., Jalava J., Kotilainen P., Jousimies-Somer H., Siitonen A. \& Huovinen P. 2002. gyrA polymorphism in Campylobacter jejuni: Detection of gyrA mutations in 162 C. jejuni isolates by Single-Strand Conformation Polymorphism and DNA sequencing. Antimicrob. Agents Chemother. 46:2644-2647.

Hall T.A. 1999. BioEdit: a user-friendly biological sequence alignment editor and analysis program for Windows 95/98/NT. Nucl. Acids. Symp. Ser. 41:95-98.

Harmon K.M., Ransom G.M. \& Wesley I.V. 1997. Differentiation of Campylobacter jejuni and Campylobacter coli by polymerase chain reaction. Mol. Cell Prob. 11:195-200.

Hermans D., Van Deun K., Messens W., Martel A., Van Immerseel F., Haesebrouck F., Rasschaert G., Heyndrickx M. \& Pasmans F. 2011. Campylobacter control in poultry by current intervention measures ineffective: Urgent need for intensified fundamental research. Vet. Microbiol. 152:219-228.

Hermans D., Frank Pasmans F., Winy Messens W., An Martel A., Filip Van Immerseel F., Geertrui Rasschaert G., Marc Heyndrickx M., Kim Van Deun K. \& Freddy Haesebrouck F. 2012. Poultry as a host for the zoonotic pathogen Campylobacter jejuni. Vector Borne Zoonotic Dis. 12(2):89-98.

Heuer O.E., Pedersen K., Andersen J.S. \& Madsen M. 2001. Prevalence and antimicrobial susceptibility of thermophilic Campylobacter in organic and conventional broiler flocks. Lett. Appl. Microbiol. 33:269-274.

Hoogenboom L.A.P., Bokhorst J.G., Northolt M.D., van de Vijver L.P.L., Broex N.J.G., Mevius D.J., Meijs J.A.C. \& Van der Roest J. 2008. Contaminants and microorganisms in Dutch organic food products: a comparison with conventional products. Food Addit. Contam. A 25:1195-1207.

Humphrey T.J., Jorgensen F., Frost J.A., Wadda H., Domingue G., Elviss N.C., Griggs D.J. \& Piddock L.J.V. 2005. Prevalence and subtypes of ciprofloxacin-resistant Campylobacter spp. in commercial poultry flocks before, during and after treatment with fluoroquinolones. Antimicrob. Agents Chemother. 49(2):690-698.

Hungaro H.M., Mendonça R.C.S., Rosa V.O., Badaró A.C.L., Moreira M.A.S. \& Chaves J.B.P. 2015. Low contamination of Campylobacter spp. on chicken carcasses in Minas Gerais state, Brazil: molecular characterization and antimicrobial resistance. Food Control 51:15-22.

Idowu O.R., Peggins J.O., Cullison R. \& Von Bredow J. 2010. Comparative pharmacokinetics of enrofloxacin and ciprofloxacin in lactating dairy cows and beef steers following intravenous administration of enrofloxacin. Res. Vet. Sci. 89:230-235.

Iovine E.N.M. 2013. Resistance mechanisms in Campylobacter jejuni. Virulence 4(3):230-240.

Jeon B., Wang Y., Hao H., Barton Y. \& Zhang Q. 2011. Contribution of CmeG to antibiotic and oxidative stress resistance in Campylobacter jejuni. J. Antimicrob. Chemother. 66:79-85.

Joshua G.W.P., Guthrie-Irons C., Karlyshev A.V. \& Wren B.W. 2006. Biofilm formation in Campylobacter jejuni. Microbiology 152:387-396.

Luangtongkum T., Morishita T.Y., Ison A.J., Huang S., McDermott P.F. \& Zhang Q. 2006. Effect of conventional and organic production practices on the prevalence and antimicrobial resistance of Campylobacter spp. in poultry. Appl. Environ. Microbiol. 72:3600-3607.

Machinski Junior M., Benini A., Netto D.P., Nunes M.P., Vedovello Filho D., Benatto A., Scucato E.S., Machado E., Belmonte I.L., Alberton M., Lopes M.O. \& Bosquiroli S.L. 2005. Medicamentos Veterinários Utilizados na Avicultura de Postura no Estado do Paraná. Relatório Anual do PAMvet. 24p. 
Možina S.S., Kurinčič M., Klančnik A. \& Mavri A. 2011. Campylobacter and its multi-resistance in the food chain. Trends Food Sci. Technol. 22:91-98.

Niederer L., Kuhnert P., Egger R., Büttner S., Hächler H. \& Korvzak B.M. 2011. Genotypes and antibiotic resistances of Campylobacter jejuni and Campylobacter coli isolates from domestic and travel-associated human cases. Appl. Environ. Microbiol. 78(1):288-291.

Norström M., Hofshagen M., Stavnes T., Schau J., Lassen J. \& Kruse H. 2007. Antimicrobial resistance in Campylobacter jejuni from broilers and broiler house environments in Norway. J. Food Prot. 70(3):736-738.

Obeng A.S., Rickard H., Sexton M., Pang Y., Peng H. \& Barton M. 2012. Antimicrobial susceptibilities and resistance genes in Campylobacter strains isolated from poultry and pigs in Australia. J. Appl. Microbiol. 113:294-307.

Overbeke I.V., Duchateau L., Zutter L.D., Albers G. \& Ducatelle R. 2006. A comparison survey of organic and conventional broiler chickens for infectious agents affecting health and food safety. Avian Dis. 50:196-200.

Oyofo B.A., Thornton S.A., Burr D.H., Pavlovskis O.R. \& Guerry P. 1992. Specific detection of Campylobacter jejuni and Campylobacter coli by using polymerase chain reaction. J. Clin. Microbiol. 30:2613-2619.

Parkhill J., Wren B.W., Mungall K., Ketley J.M., Churcher C., Basham D., Chillingworth T., Davies R.M., Feltwell T. \& Holroyd S. 2000. The genome sequence of the food-borne pathogen Campylobacter jejuni reveals hypervariable sequences. Nature 403:665-668.

Piddock L.J.V., Ricci V., Pumbwe L., Everett M.J. \& Griggs D.J. 2003. Fluoroquinolone resistance in Campylobacter species from man and animals: detection of mutations in topoisomerase genes. J. Antimicrob. Chemother. 51:19-26.

Price L.B., Johnson E., Vailes R. \& Silbergeld E. 2005. Fluoroquinoloneresistant Campylobacter isolates from conventional and antibiotic-free chicken products. Environ. Health Perspect. 113:557-560.

Qin S.S., Wu C.M., Wang Y., Jeon B., Shen Z.Q., Wang Y., Zhang Q. \& Shen J.Z. 2011. Antimicrobial resistance in Campylobacter coli isolated from pigs in two provinces of China. Int. J. Food Microbiol. 146(1):94-98.

Reeser R.J., Medler R.T., Billington S.J., Jost B.H. \& Joens L.A. 2007.Characterization of Campylobacter jejuni biofilms under defined growth conditions. Appl. Environ. Microbiol. 73(6):1908-1913.
Reuter M., Mallett A., Pearson B.M. \& van Vliet A.H.M. 2010. Biofilm formation by Campylobacter jejuni is increased under aerobic conditions. Appl. Environ. Microbiol. 76(7):2122-2128.

Rosenquist H., Boysen L., Krogh A.L., Jensen A.N. \& Nauta M. 2013. Campylobacter contamination and the relative risk of illness from organic broiler meat in comparison with conventional broiler meat. Int. J. Food. Microbiol. 162:226-230.

Soonthornchaikul N., Garelick H., Jones H., Jacobs J., Ball D. \& Choudhury M. 2006. Resistance to three antimicrobial agents of Campylobacter isolated from organically- and intensively-reared chickens purchased from retail outlets. Int. J. Antimicrob. Agents 27:125-130.

Tamura K., Stecher G., Peterson D., Filipski A. \& Kumar S. 2013. MEGA6: Molecular Evolutionary Genetics Analysis Version 6.0. Mol. Biol. Evol. 30:2725-2729.

Wagenaar J.A., French N.P. \& Havelaar A.H. 2013. Preventing Campylobacter at the source: why is it so difficult? Clin. Infect. Dis. 57(11):16001606.

Wang Y., Huang W.M. \& Taylor D.E. 1993. Cloning and nucleotide sequence of the Campylobacter jejuni gyrA gene and characterization of quinolone resistance mutations. Antimicrob. Agents Chemother. 37:457-463.

Wang Y., Chan J.P., Yeh K., Chang C., Hsuan S., Hsieh Y., Chang Y., Lai T., Lin W. \& Chen T. 2010. Molecular characterization of enrofloxacin resistant Actinobacillus pleuropneumoniae isolates. Vet. Microbiol. 142:309-312.

Wieczorek K. \& Osek J. 2013. Antimicrobial resistance mechanisms among Campylobacter. BioMed Res. Int. J. 13:1-12.

Wilson D.L., Abner S.R., Newman T.C., Mansfield L.S. \& Linz J.E. 2000. Identification of ciprofloxacin-resistant Campylobacter jejuni by use of a fluorogenic PCR assay. J. Clin. Microbiol. 38:3971-3978.

Winters D.K. \& Slavik M.F. 1995. Evaluation of a PCR based assay for specific detection of Campylobacter jejuni in chicken washes. Mol. Cell Prob. 9:307-310.

Yang M., Sahin O., Lin J. \& Zhang Q. 2006. Role of the CmeABC efflux pump in the emergence of fluoroquinolone-resistant Campylobacter under selection pressure. J. Antimicrob. Chemother. 58:1154-1159. 